\title{
Antibiotic susceptibility profiles among Campylobacter isolates obtained from international travelers between 2007 and 2014
}

\author{
A. Post ${ }^{1}$ (D) D. Martiny ${ }^{2,3,4} \cdot$ N. van Waterschoot ${ }^{5} \cdot$ M. Hallin $^{2,6} \cdot$ U. Maniewski $^{1}$.

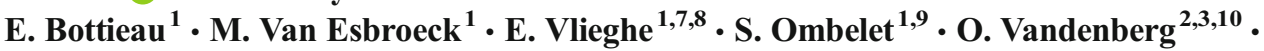 \\ J. Jacobs ${ }^{1,9}$
}

Received: 30 March 2017 / Accepted: 29 May 2017 /Published online: 17 June 2017

(C) The Author(s) 2017. This article is an open access publication

\begin{abstract}
Campylobacter infection is a common cause of diarrhea among international travelers. We studied antibiotic resistance patterns among Campylobacter isolates obtained from international travelers according to travel destination. Three collections of isolates obtained from international travelers between 2007 and 2014 (Institute of Tropical Medicine, the "Laboratoire Hospitalier Universitaire de Bruxelles "and the Belgian National Reference Centre for Campylobacter) were used. Isolates were tested for minimal inhibitory concentration (MIC) values (E-test macromethod) for fluoroquinolones, macrolides, tetracyclines, amoxicillinclavulanic acid, and meropenem. Single isolates from 261 travelers were available; median (IQR) age was 25.4 (4-42) years, $85.8 \%$ were symptomatic (information for 224 patients available). Overall resistance to ciprofloxacin was $60.9 \%$, ranging from $50.8 \%$ in Africa to $75.0 \%$ in Asia. Resistance to erythromycin was $4.6 \%$, with the highest rate observed for Southern Asia (15.2\%, seven isolates, six of them recovered from patients returning from India). A total of 126 isolates
\end{abstract}

Electronic supplementary material The online version of this article (doi:10.1007/s10096-017-3032-6) contains supplementary material, which is available to authorized users.

A. Post

annelies.post@gmail.com

1 Department of Clinical Sciences, Institute of Tropical Medicine, Nationalestraat 155, 2000 Antwerp, Belgium

2 National Reference Centre for Campylobacter, CHU Saint-Pierre, Brussels, Belgium

3 Department of Microbiology, LHUB-ULB, Pôle Hospitalier Universitaire de Bruxelles, Brussels, Belgium

4 Faculté de Médecine et Pharmacie, Université de Mons, Mons, Belgium
(48.3\%) were resistant to tetracycline. No resistance to amoxicillin-clavulanic acid or meropenem was detected. Ciprofloxacin resistance tended to increase over time (53.9\% in 2007 versus $72.2 \%$ in 2014), erythromycin resistance remained stable (median annual resistance 4.2\%). Most (86.2\%) ciprofloxacin-resistant isolates had MIC values $\geq 32 \mathrm{mg} / \mathrm{l}$, and all erythromycin-resistant isolates had MIC values $\geq 256 \mathrm{mg} / \mathrm{l}$. Co-resistance to ciprofloxacin and erythromycin was observed in 11 (4.2\%) isolates, seven of which came from Southern Asia. Among all regions of travel, more than half of Campylobacter isolates were resistant to ciprofloxacin. Overall resistance to erythromycin was below 5\% but reached $15.2 \%$ in Southern Asia.

\section{Introduction}

Campylobacter spp., in particular Campylobacter jejuni and Campylobacter coli, are the most common cause of

5 Faculty of Health, Medicine and Life Sciences, Maastricht University, Maastricht, the Netherlands

6 Department of Molecular Biology, LHUB-ULB, Pôle Hospitalier Universitaire de Bruxelles, Brussels, Belgium

7 Department of General Internal Medicine, Infectious Diseases and Tropical Medicine, University Hospital Antwerp, Antwerp, Belgium

8 University of Antwerp, Antwerp, Belgium

9 Department of Microbiology and Immunology, KU Leuven, Leuven, Belgium

10 Center for Environmental Health and Occupational Health, School of Public Health, Université Libre de Bruxelles, Brussels, Belgium 
bacterial enteritis in humans worldwide. Typically Campylobacter infection causes self-limiting diarrhea with or without fever [1]. The severity of symptoms may require antibiotic treatment; most commonly erythromycin or fluoroquinolones [1-3]. In industrialized countries, travel-related campylobacteriosis represents an important subset of all reported cases, ranging from $13 \%$ in the USA [4] to $20 \%$ in Scandinavia $[5,6]$.

The objective of the present study was to update antibiotic resistance rates among Campylobacter isolates obtained from international travelers between 2007 and 2014, compared to a previous study over the time period 1994-2006 [7].

\section{Methods}

\section{Design}

Three collections of human intestinal Campylobacter isolates consecutively obtained from international travelers were tested. A first collection one ( $n=194$ ) was obtained from the posttravel policlinic at the Institute of Tropical Medicine (ITM) Antwerp. The second collection $(n=84)$ consisted of clinical isolates obtained from the Laboratoire de la Porte de Hal (LHUB-ULB), Brussels. The third collection $(n=37)$ formed part of a National Survey in Belgium, performed by the National Reference Centre for Campylobacter in Brussels (NRC). Methods of isolation and identification of isolates differed between collections. A detailed description of patient population, study period, and methods used for each collection can be found in Supplement 1. Only patients with a history of travel ( $\leq 2$ weeks if symptomatic and $\leq 8$ weeks if asymptomatic) prior to consultation were included in this study, and only the first isolate per patient was considered. For the purpose of this study, subcultures of all isolates obtained at LHUB-ULB or NRC from patients with a reported travel history were shipped to ITM and stored on Microbank at $-80{ }^{\circ} \mathrm{C}$ pending testing.

\section{Antibiotic susceptibility testing}

Upon retrieval from storage isolates were subcultured onto Columbia agar with $5 \%$ sheep blood and incubated at $42{ }^{\circ} \mathrm{C}$ for $24 \mathrm{~h}$. Antibiotic susceptibility testing was performed in batch using Mueller Hinton agar with 5\% sheep blood and incubated in a microaerophilic atmosphere at $42{ }^{\circ} \mathrm{C}$ for 24 to $48 \mathrm{~h}$. Minimal inhibitory concentration (MIC) values for ciprofloxacin, levofloxacin, azithromycin, erythromycin, tetracycline, doxycycline, amoxicillin-clavulanic acid and meropenem were determined by E-test macromethod (bioMérieux, Marcyl'Etoile, France). This panel was chosen in accordance with EUCAST guidelines and recommendations of the European Centre for Disease Prevention and Control (ECDC) [8]. Breakpoints are summarized in Table 1. EUCAST does not provide breakpoints for amoxicillinclavulanic acid and levofloxacin. For amoxicillinclavulanic acid, the EUCAST breakpoints for Enterobacteriaceae were used. For levofloxacin EUCAST breakpoints for Enterobacteriaceae are the same as those proposed for Helicobacter pylori, which is often considered as a Campylobacter-related organism [9]. For meropenem, the interpretation criteria as recommended by the Clinical and Laboratory Standards Institute
Table 1 Panel of tested antibiotics and corresponding breakpoints

\begin{tabular}{|c|c|c|}
\hline Antibiotic & $\begin{array}{l}\text { Breakpoint* } \\
(\mathrm{mg} / \mathrm{l})\end{array}$ & Reference guideline \\
\hline Ciprofloxacin & $\leq 0.5$ & EUCAST guideline for Campylobacter \\
\hline Levofloxacin & $\leq 1$ & EUCAST guideline for Helicobacter pylori \\
\hline Erythromycin & & EUCAST guideline for Campylobacter \\
\hline C. jejuni & $\leq 4$ & \\
\hline C. coli & $\leq 8$ & \\
\hline Azithromycin & None & $\begin{array}{l}\text { EUCAST and CLSI mention to use results for erythromycin to } \\
\text { determine susceptibility to azithromycin }\end{array}$ \\
\hline Tetracycline & $\leq 2$ & EUCAST guideline for Campylobacter \\
\hline Doxycycline & None & $\begin{array}{l}\text { No breakpoints available, EUCAST and CLSI mention to use results } \\
\text { for tetracycline to determine susceptibility to doxycycline }\end{array}$ \\
\hline $\begin{array}{l}\text { Amoxicillin/clavulanic } \\
\text { acid }\end{array}$ & $\leq 8$ & EUCAST guideline for Enterobacteriaceae \\
\hline Meropenem & $<16$ & $\begin{array}{l}\text { No breakpoints available, EUCAST mention to use CLSI guideline } \\
\text { for Campylobacter }\end{array}$ \\
\hline
\end{tabular}

*S and $\mathrm{R}$ breakpoints are identical

Guidelines: EUCAST guideline version 7.0, 2017 / CLSI guideline M100-S26, 2016 
(CLSI) were used [8, 10]. Quality control for antibiotic susceptibility was done according to manufacturer's guidelines.

\section{Demographic and clinical data}

Travel destinations were grouped in regions and continents according to the United Nations geoscheme [11]. Demographic and clinical data including age, gender, fever, diarrhea, and duration of symptoms were retrieved from patient files or from forms filled by participating labs and recorded in a protected database. Data were entered into an encrypted database for analysis.

\section{Statistical analysis}

Proportions of resistance rates over time and for different geographical regions were assessed for significance using the Pearson's chi-square test $\left(\mathrm{X}^{2}\right.$-test) or Fisher's exact test. Trends over time were assessed using the Mantel-Haenszel extension of the chi-square test for trends. A $p$-value $<0.05$ was considered significant. For convenience, differences between the three collections are only discussed separately in the text if they are significant.

\section{Ethical approval}

Ethical approval was obtained at the respective ethics committees for both study sites; the Internal Review Board and the University Hospital of Antwerp for ITM and Ethical Committee of CHU Saint Pierre.

\section{Results}

Of the 267 available isolates, six were excluded because information about travel destination was not available, resulting in a total of 261 isolates recovered from 261 patients. At ITM, 142 isolates were available for analysis, representing $73.2 \%$ of all 194 non-duplicate isolates obtained at this site during the study period: non-available isolates included those that had not been stored $(n=41)$ and those that did not grow upon retrieval $(n=11)$. The LHUB-ULB collection consisted of 83 available isolates, representing $98.8 \%$ of 84 isolates from

Table 2 Travel destinations of patients with a Campylobacter infection, represented per patient group. In brackets, countries with high numbers of isolates). Total number of isolates $=261$

\begin{tabular}{|c|c|c|c|c|c|}
\hline $\begin{array}{l}\text { Travel destination according } \\
\text { to UN geoscheme }\end{array}$ & $\begin{array}{l}\text { Most frequently visited countries } \\
\text { in the region (number of isolates) }\end{array}$ & $\begin{array}{l}\text { ITM, } n=142 \\
N(\%)\end{array}$ & $\begin{array}{l}\text { LHUB-ULB, } n=83 \\
N(\%)\end{array}$ & $\begin{array}{l}\text { National survey, } n=36 \\
N(\%)\end{array}$ & $\begin{array}{l}\text { Total, } n=261 \\
N(\%)\end{array}$ \\
\hline Eastern Africa & Ethiopia (12), Tanzania (5) & $26(18.3 \%)$ & $3(3.6 \%)$ & $1(2.8 \%)$ & $30(11.5 \%)$ \\
\hline Middle Africa & $\operatorname{DRC}^{1}(16)$ & $18(12.7 \%)$ & $7(8.4 \%)$ & 0 & $25(9.6 \%)$ \\
\hline Northern Africa & Morocco (22), Tunis (5) & $5(3.52 \%)$ & $24(28.9 \%)$ & $7(19.4 \%)$ & $36(13.8 \%)$ \\
\hline Southern Africa & South Africa (1) & $1(0.7 \%)$ & 0 & 0 & $1(0.4 \%)$ \\
\hline Western Africa & Burkina Faso (6), Cameroon (5) & $20(14.1 \%)$ & $8(9.6 \%)$ & 0 & $28(10.7 \%)$ \\
\hline Africa total & & $70(49.3 \%)$ & $42(50.6 \%)$ & $8(22.2 \%)$ & $120(46.0 \%)$ \\
\hline $\begin{array}{l}\text { Caribbean and Latin } \\
\text { America }\end{array}$ & Cuba (1) & $2(1.4 \%)$ & $1(1.2 \%)$ & 0 & $3(1.1 \%)$ \\
\hline Central America & Nicaragua (1) & $1(0.7 \%)$ & 0 & 0 & $1(0.4 \%)$ \\
\hline South America & Peru (9) & $13(9.2 \%)$ & $2(2.4 \%)$ & 0 & $15(5.7 \%)$ \\
\hline Northern America & $\mathrm{USA}^{2}(1)$ & 0 & 0 & $1(2.8 \%)$ & $1(0.4 \%)$ \\
\hline America total & & $16(11.3 \%)$ & $3(3.6 \%)$ & $1(2.8 \%)$ & $20(7.7 \%)$ \\
\hline Eastern Asia & China (1) & $1(0.7 \%)$ & 0 & 0 & $1(0.4 \%)$ \\
\hline Southern Asia & India (31), Nepal (5), Pakistan (5) & $31(21.8 \%)$ & $13(15.7 \%)$ & $2(5.6 \%)$ & $46(17.6 \%)$ \\
\hline Southeastern Asia & Indonesia (10) & $21(14.8 \%)$ & $1(1.2 \%)$ & $3(8.3 \%)$ & $25(9.6 \%)$ \\
\hline Western Asia & Turkey (7) & 0 & $2(2.4 \%)$ & $6(16.7 \%)$ & $8(3.1 \%)$ \\
\hline Asia total & & $53(37.3 \%)$ & $16(19.3 \%)$ & $11(30.6 \%)$ & $80(30.7 \%)$ \\
\hline Eastern Europe & Poland (3), Romania (2) & 0 & $6(7.2 \%)$ & $2(5.6 \%)$ & $8(3.1 \%)$ \\
\hline Southern Europe & Spain (8) & $2(1.4 \%)$ & $9(10.8 \%)$ & $6(16.7 \%)$ & $17(6.5 \%)$ \\
\hline Western Europe & France (10) & $1(0.7 \%)$ & $7(8.4 \%)$ & $8(22.2 \%)$ & $16(6.1 \%)$ \\
\hline Europe total & & $3(2.8 \%)$ & $22(26.5 \%)$ & $16(44.4 \%)$ & $41(15.7 \%)$ \\
\hline
\end{tabular}

1. DRC: Democratic Republic of the Congo

2. USA: United States of America 
patients with a recent travel history. In addition, 36 (97.3\% of 37) isolates from participants with a recent travel history were available through the National Survey. $C$. jejuni was most prevalent $(n=230,88.1 \%)$, followed by $C$. coli $(n=22$, 8.4\%); nine isolates (3.4\%) were not identified beyond the genus level. There was no significant difference in geographical distribution between the different Campylobacter species.

\section{Patient characteristics and geographical origin of isolates}

The median (IQR) age of the entire cohort was $25.4(4-42)$ years; $50.4 \%$ of patients were male. Median ages differed between the three collections: $28.5,2.5$, and 49.0 years for the ITM, LHUB-ULB and National Survey collections respectively, with $8.4 \%, 58.3 \%$, and $16.7 \%$ of patients younger than 5 years respectively. Complete data about a history of diarrhea and fever were available for 243 (93.1\%) and 249 (95.4\%) patients respectively: most $(224,85.8 \%)$ suffered from diarrhea, whereas 88 (33.7\%) reported fever and 84 (31.8\%) reported both. Seventeen patients were asymptomatic; in these participants, the isolate was obtained during routine post-travel consultation. Table 2 presents the distribution of travel destinations

Table 3 Resistance rates of Campylobacter isolates represented by travel region defined according to United Nations geoscheme. Intermediate susceptible results are grouped together with resistant results

\begin{tabular}{|c|c|c|c|c|}
\hline Region & $N$ & Ciprofloxacin & Erythromycin & Tetracycline \\
\hline Eastern Africa & 30 & $13(43.3 \%)$ & 0 & $17(56.7 \%)$ \\
\hline Middle Africa & 25 & $13(52.0 \%)$ & $1(4.0 \%)$ & $12(48.0 \%)$ \\
\hline Northern Africa & 36 & $24(66.7 \%)$ & $1(2.8 \%)$ & $18(50.0 \%)$ \\
\hline Southern Africa & 1 & 0 & 0 & 0 \\
\hline Western Africa & 28 & $11(39.3 \%)$ & $1(3.6 \%)$ & $9(32.1 \%)$ \\
\hline Africa total & 120 & $61(50.8 \%)$ & $3(2.5 \%)$ & $56(46.7 \%)$ \\
\hline Caribbean & 3 & 1 & 1 & 0 \\
\hline Central America & 1 & 1 & 0 & 1 \\
\hline South America & 15 & $12(80 \%)$ & 0 & $9(60.0 \%)$ \\
\hline Northern America & 1 & 1 & 0 & 1 \\
\hline America total & 20 & $15(75.0 \%)$ & $1(5.0 \%)$ & $11(55.0 \%)$ \\
\hline Eastern Asia & 1 & 0 & 0 & 0 \\
\hline Southern Asia & 46 & $35(76.1 \%)$ & $7(15.2 \%)$ & $19(41.3 \%)$ \\
\hline Southeastern Asia & 25 & $17(68.0 \%)$ & $1(4.0 \%)$ & $14(56.0 \%)$ \\
\hline Western Asia & 8 & 8 & 0 & 4 \\
\hline Asia total & 80 & $60(75.0 \%)$ & $8(10.0 \%)$ & $37(46.3 \%)$ \\
\hline Eastern Europe & 8 & 4 & 0 & 6 \\
\hline Southern Europe & 17 & $8(47.1 \%)$ & 0 & $6(35.3 \%)$ \\
\hline Western Europe & 16 & $11(68.8 \%)$ & $1(6.3 \%)$ & $10(62.5 \%)$ \\
\hline Europe total & 41 & $23(56.1 \%)$ & $1(2.4 \%)$ & $22(53.7 \%)$ \\
\hline Total & 261 & $159(60.9 \%)$ & $12(4.6 \%)$ & $126(48.3 \%)$ \\
\hline
\end{tabular}

If the total number of isolates for a particular region is $<10$, the percentage of resistance is not mentioned. of the patients with Campylobacter infection, grouped according to the United Nations geoscheme [11]. More than threequarters of travelers had returned from Africa (46.0\%) or Asia $(30.7 \%)$.

\section{Antibiotic resistance: Geographic distribution and evolution over time (Table 3)}

A total of 77 isolates (29.5\%) were susceptible to all antibiotics tested, of which 43 isolates (55.8\%) were obtained from Africa. Overall resistance to ciprofloxacin was $60.9 \%$, ranging from $50.8 \%$ in Africa to $75.0 \%$ in Asia. Lowest resistance rates although consistently above $33 \%$ of isolates - were observed for Eastern, Western, and Middle Africa. Overall resistance to erythromycin was $4.6 \%$, and ranged from $2.4 \%$ in Europe to $10.0 \%$ in Asia. The highest rate was observed for Southern Asia $(15.2 \%)$, with 6/7 resistant isolates recovered from India. A total of 126 isolates $(48.3 \%$ ) were resistant to tetracycline, with small variations between regions. There was no resistance to amoxicillin - clavulanic acid or meropenem. During the study period, ciprofloxacin resistance rate was higher in $2014(72.2 \%)$ than in 2007 (53.9\%). When comparing the present ciprofloxacin resistance rate to that of 2001-2006 as tested in our previous study [7],we find a significant increase over time $(p=0.03)$.

For erythromycin, no increase over time was noted (median annual resistance $4.2 \%$, Fig. 1). Compared to the aforementioned study [7], overall resistance against erythromycin was slightly, but not statistically higher in the present study compared to the 2001-2006 period (4.6\% versus $2.7 \%, p=0.2$ ).

\section{Co-resistance and MIC values}

Resistance rates for levofloxacin and ciprofloxacin were identical $(60.9 \%, 159$ isolates) with complete overlap. Most (86.2\%) ciprofloxacin-resistant isolates (86.2\%) had MIC values

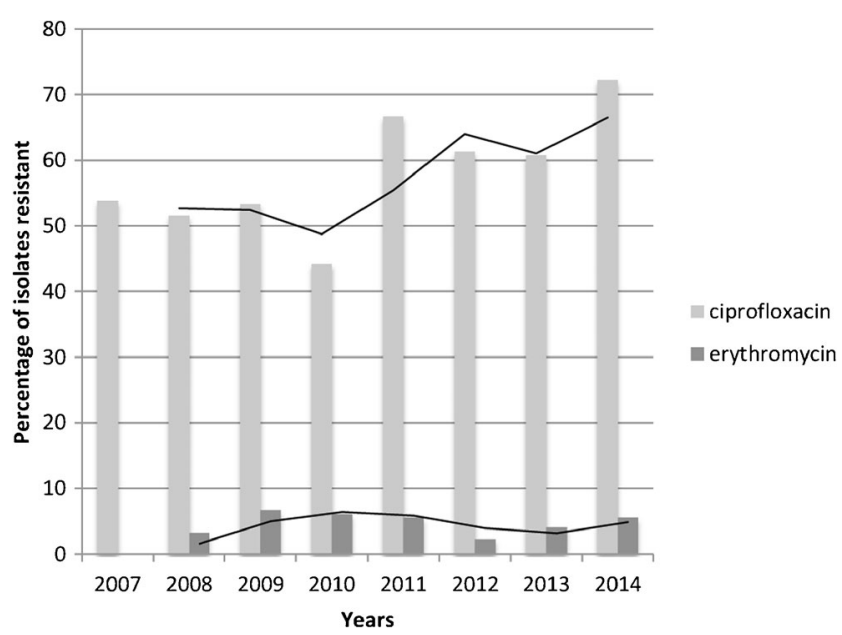

Fig. 1 Evolution of ciprofloxacin and erythromycin resistance (expressed as \% resistance) over the time period 2007-2014 
$\geq 32 \mathrm{mg} / 1$ (Fig. 2). Levofloxacin-resistant strains displayed two subgroups; isolates with lower MIC values (around $6 \mathrm{mg} / \mathrm{l}$, $60.0 \%$ of resistant isolates) and isolates with high MIC values ( $\geq 32 \mathrm{mg} / \mathrm{l}, 34.4 \%$ of resistant isolates). Resistance to erythromycin and azithromycin was seen among 12 isolates (4.6\%); MIC values were in complete overlap, and all isolates displayed high-level resistance $(\geq 256 \mathrm{mg} / \mathrm{l})$ for both antibiotics. Resistance rates to erythromycin among Campylobacter coli tended to be lower than to those for Campylobacter jejuni; $9.1 \%$ and $4.3 \%$ respectively ( $p=0.3$ ). Resistance rates to ciprofloxacin were similar for both species. Co-resistance to ciprofloxacin and erythromycin was observed in $11(4.2 \%)$ isolates, seven of which came from Southern Asia (six from India, one from Nepal).

\begin{tabular}{|c|c|c|c|c|c|}
\hline \multicolumn{3}{|c|}{ MIC ciprofloxacin } & \multicolumn{3}{|c|}{ MIC erythromycin } \\
\hline $\begin{array}{l}\text { MIC- } \\
\text { value }\end{array}$ & \multicolumn{2}{|c|}{$\begin{array}{l}\text { Nrs of } \\
\text { isolates }\end{array}$} & $\begin{array}{l}\text { MIC- } \\
\text { value }\end{array}$ & \multicolumn{2}{|c|}{$\begin{array}{l}\text { Nrs of } \\
\text { isolates }\end{array}$} \\
\hline 0.004 & \multicolumn{2}{|l|}{0} & 0.016 & \multicolumn{2}{|l|}{0} \\
\hline 0.006 & \multicolumn{2}{|l|}{0} & 0.023 & \multicolumn{2}{|l|}{0} \\
\hline 0.008 & \multicolumn{2}{|l|}{0} & 0.032 & \multicolumn{2}{|l|}{1} \\
\hline 0.012 & \multicolumn{2}{|l|}{0} & 0.047 & \multicolumn{2}{|l|}{0} \\
\hline 0.016 & \multicolumn{2}{|l|}{2} & 0.064 & \multicolumn{2}{|l|}{1} \\
\hline 0.023 & \multicolumn{2}{|l|}{4} & 0.094 & \multicolumn{2}{|l|}{1} \\
\hline 0.032 & \multicolumn{2}{|l|}{9} & 0.125 & \multicolumn{2}{|l|}{3} \\
\hline 0.047 & \multicolumn{2}{|l|}{16} & 0.19 & \multicolumn{2}{|l|}{2} \\
\hline 0.064 & \multicolumn{2}{|l|}{35} & 0.25 & \multicolumn{2}{|l|}{15} \\
\hline 0.094 & \multicolumn{2}{|l|}{12} & 0.38 & \multicolumn{2}{|l|}{24} \\
\hline 0.125 & \multicolumn{2}{|l|}{19} & 0.5 & \multicolumn{2}{|l|}{60} \\
\hline 0.19 & \multicolumn{2}{|l|}{3} & 0.75 & 48 & \multirow[t]{3}{*}{ MIC50 } \\
\hline 0.25 & \multicolumn{2}{|l|}{1} & 1 & \multirow{2}{*}{$\begin{array}{l}49 \\
23\end{array}$} & \\
\hline 0.38 & \multicolumn{2}{|l|}{1} & 1.5 & & \\
\hline 0.5 & 0 & $\mathrm{R}$ & 2 & 14 & MIC90 \\
\hline 0.75 & 0 & & 3 & 5 & \\
\hline 1 & 0 & & 4 & 3 & $\mathrm{R}^{*}$ \\
\hline 1.5 & 2 & & 6 & 0 & \\
\hline 2 & 0 & & 8 & 0 & $\mathrm{R}^{+}$ \\
\hline 3 & 2 & & 12 & 0 & \\
\hline 4 & 2 & & 16 & 0 & \\
\hline 6 & 5 & & 24 & 0 & \\
\hline 8 & 2 & & 32 & 0 & \\
\hline 12 & 3 & & 48 & 0 & \\
\hline 16 & 5 & & 64 & 0 & \\
\hline 24 & 1 & & 96 & 0 & \\
\hline$\geq 32$ & 137 & M50 & 128 & 0 & \\
\hline & & & 192 & 0 & \\
\hline & & & $\geq 256$ & 12 & \\
\hline
\end{tabular}

* Resistance breakpoint for Campylobacter jejuni

+ Resistance breakpoint for Campylobacter coli

Fig. 2 MIC-value distribution including MIC50 and MIC90 for ciprofloxacin and erythromycin among Campylobacter isolates, recovered from international travelers. $R$ displays resistance breakpoint (see Table 1)

\section{Discussion}

In this study, we assessed antimicrobial resistance patterns among Campylobacter isolates obtained from stool samples from international travelers between 2007 and 2014.

The isolates used in this study came from collections with different travelers' profiles and travel destinations: ITM serves a large patient population returning or originating from subSaharan Africa, while the LHUB-ULB serves hospitals that provide healthcare to a large population of Northern African origin. The National Survey was conducted during the holiday season and is likely to be the best representation of the Belgian tourists' destinations (Europe and Asia). Most travelers were symptomatic.

\section{Comparison to literature}

Overall, more than half of Campylobacter isolates were resistant to ciprofloxacin, with highest rates observed for Asia and the Americas. Data about resistance to fluoroquinolones among Campylobacter spp. in humans varies, with levels as high as $93.1 \%$ in China [12] to $6.6 \%$ in Finland [6]. The increase in ciprofloxacin resistance over the present study period is in line with what we observed previously [7], and was mainly accounted for by a steady increase in resistance among the African regions.

More than $80 \%$ of ciprofloxacin-resistant isolates had MIC values $\geq 32 \mathrm{mg} / \mathrm{l}$. As $32 \mathrm{mg} / \mathrm{l}$ is the upper value at which the Etest is truncated, the actual MIC value of these isolates was not assessed. It should be remembered that peak concentration of fluoroquinolones in feces largely exceeds those reached in blood after standard-dose therapy. This can explain why fluoroquinolone treatment of campylobacteriosis may result in clinical cure despite apparent resistance of the isolate (23). Molecular mechanisms for fluoroquinolone resistance (not assessed in the current collection) include point mutations in the genes encoding DNA gyrase (gyrA) and an efflux system mechanism [13-15].

Resistance to macrolides as assessed in the present study was particularly high in Asia, with a resistance rate of $15.2 \%$ in Southern Asia. These results confirm the high level of resistance previously reported in a Pakistani urban environment in 2012, where $27.0 \%$ of $C$. jejuni human isolates were resistant to erythromycin [16]. Resistance to macrolides has been reported to be higher among C. coli (up to $66 \%$ [17]) compared to $C$. jejuni (less than 10\%), but this was not confirmed in the present study, possibly because of low numbers of $C$. coli isolates.

The $15.2 \%$ resistance rate among isolates from Southern Asia, as well as the occurrence of combined ciprofloxacinerythromycin resistance in India and Nepal (representing 7/11 co-resistant isolates), argue for caution and continued 
surveillance. Resistant isolates displayed high-level resistance for both antibiotics (MIC value $\geq 256 \mathrm{mg} / \mathrm{l}$ ). This is indicative of resistance conferred by point mutations in the domain $\mathrm{V}$ in the 23S ribosomal RNA gene [17]. Our results confirm that azithromycin susceptibility can indeed be derived from erythromycin results.

In the present collection, there was no resistance against amoxicillin-clavulanic acid. Data about resistance levels to amoxicillin-clavulanic acid are scarce, as it is currently not a treatment of choice [18]. Resistance to carbapenems was not observed in the present study, but has been reported anecdotally among Campylobacter fetus [19].

\section{Limitations}

The study was conducted with different isolate collections, originating from different time periods and various methods of isolation and identification. Additionally, the numbers of isolates varied between years, and 32 isolates obtained at ITM between October 2010 and August 2011 had not been stored. Detailed and complete clinical data (symptoms, duration of illness) were only available for a subset of patients. Finally, the number of isolates obtained from some regions were too small to draw reliable conclusions.

Conversely, combining different isolate collections made it possible to cover different profiles and destinations of travelers; the resulting numbers of isolates were sufficient to generate a geographic picture as well as an over-time evolution. In that regard, the study can be considered as representative for travel medicine, and in addition provides proxy antibiotic surveillance data from regions in the world where microbiological surveillance is scarce.

\section{Clinical implications}

Antibiotic treatment for Campylobacter diarrhea is rarely indicated, as it is self-limiting and will usually cure within 57 days of onset of symptoms. In the case of patients whose symptoms are severe or persistent, immunocompromised patients, and in those with extra-intestinal infections, antibiotic treatment is needed. The present resistance rates confirm that, if indicated, azithromycin is the antibiotic of choice in travelassociated diarrhea contracted worldwide [7].

In conclusion, more than half of Campylobacter isolates recovered from symptomatic travelers returning from all regions were resistant to ciprofloxacin, precluding its use in the treatment of Campylobacter diarrhea. Overall resistance to erythromycin was below 5\%, but as high as $15.2 \%$ in Southern Asia. The World Health Organization recently ranked fluoroquinolone-resistant Campylobacter spp. as a high-priority pathogen for the development of new antibiotics [20]. This necessity is highlighted by the increase in combined fluoroquinolone and macrolide resistance in Southern Asia, as demonstrated in this study. This study furthermore underlines the importance of routine surveillance, in particular in lowresource settings, and the reinforcement of restrictions on the use of antibiotics in humans and the animal industry.

Acknowledgements We would like to thank Marleen Verlinden for her technical support during this study.

\section{Compliance with ethical standards}

Funding statement No funding was received for this study.

Conflict of interest The authors declare that they have no conflicts of interest.

Data availability statement All generated or analyzed data are included in this published article and the supplementary information files.

Open Access This article is distributed under the terms of the Creative Commons Attribution 4.0 International License (http:// creativecommons.org/licenses/by/4.0/), which permits unrestricted use, distribution, and reproduction in any medium, provided you give appropriate credit to the original author(s) and the source, provide a link to the Creative Commons license, and indicate if changes were made.

\section{References}

1. Kaakoush NO, Castano-Rodriguez N, Mitchell HM, Man SM (2015) Global epidemiology of campylobacter infection. Clin Microbiol Rev 28(3):687-720

2. Antimicrobial Therapy Inc. (2012-2013) Sanford guide to antimicrobial therapy. Antimicrobial Therapy Inc., Sperryville VA

3. SWAB (2014) Optimaliseren van het antibioticabeleid in Nederland. Antibiotische therapie per verwekker. Stichting Werkgroep Antibiotica Beleid (SWAB), Nijmegen, p 8

4. WHO (2012) The global view of Campylobacteriosis: report of an expert consultation. WHO, Utrecht

5. Ekdahl K, Andersson Y (2004) Regional risks and seasonality in travel-associated campylobacteriosis. BMC Infect Dis 4(1):54

6. Feodoroff B, Lauhio A, Ellstrom P, Rautelin H (2011) A nationwide study of campylobacter jejuni and campylobacter coli bacteremia in Finland over a 10-year period, 1998-2007, with special reference to clinical characteristics and antimicrobial susceptibility. Clin Infect Dis 53(8):e99-e106

7. Vlieghe ER, Jacobs JA, Van Esbroeck M, Koole O, Van Gompel A (2008) Trends of norfloxacin and erythromycin resistance of campylobacter jejuni/campylobacter coli isolates recovered from international travelers, 1994 to 2006. J Travel Med 15(6):419-425

8. European Centre for Disease Prevention and Control (2014) EU protocol for harmonised monitoring of antimicrobial resistance in human Salmonella and Campylobacter isolates, Sweden, http:// ecdc.europa.eu/en/publications/Publications/AMR-salmonellacampylobacter-protocol-monitoring.pdf. Cited Accessed; 06-092015

9. On SL (2001) Taxonomy of Campylobacter, Arcobacter, Helicobacter and related bacteria: current status, future prospects, and immediate concerns. Symposium series (Society for Applied Microbiology) 30:1s-15s 
10. CLSI (2016)M100-S26 performance standards for antimicrobial susceptibility testing; twenty-fourth informational supplement. CLSI, Wayne PA

11. United Nations (2013) Composition of macro geographical (continental) regions, geographical sub-regions, and selected economic and other groupings. UN Statistics Division

12. Zhou J, Zhang M, Yang W, Fang Y, Wang G, Hou F (2016) A seventeen-year observation of the antimicrobial susceptibility of clinical campylobacter jejuni and the molecular mechanisms of erythromycin-resistant isolates in Beijing, China. Int J Infect Dis 42:28-33

13. Griggs DJ, Johnson MM, Frost JA, Humphrey T, Jorgensen F, Piddock LJ (2005) Incidence and mechanism of ciprofloxacin resistance in campylobacter spp. isolated from commercial poultry flocks in the United Kingdom before, during, and after fluoroquinolone treatment. Antimicrob Agents Chemother 49(2):699-707

14. Iovine NM (2013) Resistance mechanisms in campylobacter jejuni. Virulence 4(3):230-240
15. Wieczorek K, Osek J (2013) Antimicrobial resistance mechanisms among campylobacter. Biomed Res Int 2013:340605

16. Siddiqui FM, Akram M, Noureen N, Noreen Z, Bokhari H (2015) Antibiotic susceptibility profiling and virulence potential of campylobacter jejuni isolates from different sources in Pakistan. Asian Pac J Trop Med 8(3):197-202

17. Engberg J, Aarestrup FM, Taylor DE, Gerner-Smidt P, Nachamkin I (2001) Quinolone and macrolide resistance in campylobacter jejuni and C. Coli: resistance mechanisms and trends in human isolates. Emerg Infect Dis 7(1):24-34

18. Abay S, Kayman T, Otlu B, Hizlisoy H, Aydin F, Ertas N (2014) Genetic diversity and antibiotic resistance profiles of campylobacter jejuni isolates from poultry and humans in Turkey. Int J Food Microbiol 178:29-38

19. Mosca A, Del Gaudio T, Miragliotta G (2010) Imipenem-resistant campylobacter fetus bloodstream infection. J Chemother 22(2):142

20. WHO (2017) WHO priority pathogens list for R\&D of new antibiotics. [Available from: http://www.who.int/medicines/publications/ global-priority-list-antibiotic-resistant-bacteria/en/] 